\title{
A Fuzzy Decision Support Model for Natural Disaster Response under Informational Uncertainty
}

\author{
Felix Wex, Information Systems Research, University of Freiburg, Germany \\ Guido Schryen, Information Systems Research, University of Regensburg, Germany \\ Dirk Neumann, Information Systems Research, University of Freiburg, Germany
}

\begin{abstract}
Coordination deficiencies have been identified after the March 2011 earthquakes in Japan in terms of scheduling and allocation of resources, with time pressure, resource shortages, and especially informational uncertainty being main challenges. We address this issue of operational emergency response in natural disaster management (NDM) by suggesting a decision support model and a Monte Carlo heuristic which account for these challenges by drawing on fuzzy set theory and fuzzy optimization.

Deriving requirements for addressing NDM situations from both practice and literature, we propose a decision model that accounts for the following phenomena: a) incidents and rescue units are spatially distributed, b) rescue units possess specific capabilities, c) processing is non-preemptive, and d) informational uncertainty occurs due to vague and linguistic specifications of data. We computationally evaluate our heuristic and benchmark the results with current best practice solutions. Our results indicate that applying the new heuristic can substantially reduce overall harm.
\end{abstract}

Keywords:

Decision Support Systems, Optimization, Coordination, Informational Uncertainty, Fuzzy Set Theory, Fuzzy Optimization 


\section{Introduction}

Natural disasters, including earthquakes, tsunamis, floods, hurricanes, and volcanic eruptions, have caused tremendous harm and continue to threaten millions of humans and various infrastructure capabilities each year. Being consistent with the terminology of the International Federation of Red Cross and Red Crescent Societies (IFRC) and the U.S. Federal Emergency Management Agency (FEMA), we use the term "disaster" in the following sense (IFRC): "A disaster is a sudden, calamitous event that seriously disrupts the functioning of a community or society and causes human, material, and economic or environmental losses that exceed the community's or society's ability to cope using its own resources." In this study, we focus on disasters based on natural disasters, rather on technological, man-made, or attack-based disasters. In contrast to disasters of the latter types, their natural counterparts are not preventable. Thus, both the actions that need to be taken before, during and after disasters and the used data are different. For example, risk management of floods and hurricanes can draw on geological data while the risk management of nuclear attacks by terrorists cannot do so.

The coordination of resources during natural disasters is characterized by a high level of informational uncertainty due to the chaotic situation, severe resource shortages, and a high demand for timely information in the presence of the disruption of infrastructure support (Chen et al., 2008). The March 2011 earthquakes near the coast of Sendai, Japan manifested these presumptions, as did the management of the succeeding nuclear disaster (Krolicki, 2011). Emergency operations centers (EOC) were confronted with the partial breakdown of information systems and transportation infrastructure. Officials had to deal with numerous incidents where more than 27,000 people were found dead or missing and some 150,000 Japanese displaced (Sanders, 2011). Actions of local commanders and rescue teams were coined by a high degree of improvisation and decentralization. The involvement of numerous, international organizations with different disaster response policies, resources, and technological infrastructures as well as capabilities led to distributed planning and implementing of response actions (Chawla, 2011). Poor communication between geographically dispersed EOCs, a lack of clear command structure and accurate data, and an immense time pressure intensified the dilemma (Deutsche Presse-Agentur, 2011; Dmitracova, 2010). Even though resource scarcity can occur, we argue that the "appropriate allocation of [spatially distributed] resources is more important (...) [and] a problem of coordination" (Comfort et al., 2004; Klingner, 2011).

The above issues reveal that the allocation of rescue units to incidents remains a challenge in effectively utilizing available resources and designing Emergency Response Systems (ERS). In practice, as told by associates of the German Federal Agency of Technical Relief (THW), assignments and schedules for resources are still derived through the application of greedy policies: for example, based on a ranking of incidents in terms of destructiveness, the most severe incidents are sequentially handled by the closest, idle rescue units (also stated by Comfort (1999)). However, this straightforward - albeit in many cases common and favorable - rule ignores estimated processing times of incidents, which may significantly affect overall casualties and harm.

When EOCs face the challenge to coordinate their rescue units they usually find a chaotic situation in which much information is inherently uncertain. For example, the severity of incidents is described in terms of linguistic terms, such as "lots of damage" or "a little fire burning". Subsequently, information on how much time rescue units need to process these incidents is vague if known at all. The chaotic situation does also not allow making precise statements on how long rescue units travel between two points of incidents as the traffic infrastructure may be severely affected. All these types of information have in common that 
the impreciseness of predictions is due to a lack of information, belief, and linguistic characterizations, which all are deemed some of the most important roots of uncertainty (Zimmermann, 2000). In the absence of statistical information and in the presence of subjective uncertainty we account for these roots of uncertainty by drawing on fuzzy set theory (Zadeh, 1965) among the many available uncertainty theories. Fuzzy set theory in emergency response situations has been stated appropriate (Altay \& Green III, 2006). This fact is particularly based on the idea that "a [fuzzy set theory based] framework provides a natural way of dealing with problems in which the source of imprecision is the absence of sharply defined criteria of class membership rather than the presence of random variables." (Zadeh, 1965)

We also argue that time is the most crucial factor during emergency response coordination and thus a proxy for harm and argue for the primary goal to minimize the sum of weighted completion times of incidents, where completion times can be defined as the duration of the occurrence of an incident until its extinction. As the literature provides some papers on decision support in emergency response situations, the purpose of our paper is to suggest a mathematical decision model for the assignment of incidents to rescue units and their scheduling under informational uncertainty, and to propose and to computationally evaluate a (Monte Carlo) solution heuristic.

This paper is an extended version of Wex et al. (2012) presented at the 2012 ISCRAM conference. The remainder of this paper is structured as follows: based on a review from scholars and interviews with practitioners, we identify requirements for a decision support model and for possible solution approaches. We then suggest a fuzzy non-linear optimization model and propose a Monte Carlo solution heuristic. We describe the computational evaluation, which attests the advantages of the suggested solution approach over a procedure which is found in practice. In the end, the paper discusses the results before it closes with a conclusion.

\section{Requirement Engineering}

We first motivate the need for centralized decision support before we derive requirements on dedicated decision support models:

A lack of centralized coordination may yield (a) deficiencies in terms of control over actions of rescue units and (b) error-prone supervision caused by inhomogeneous or duplicate commands to multi-autonomous agents with limited information about other actors' status and positions (Airy et al., 2009). When international aid organizations come and work together during a disaster, they consequently "put themselves under the control of the responsible EOC without losing their internal, autarkic command structure" (cit. THW, translated). Following the argument of Rolland et al. (2010), that congruent activities and non-interference among multiple decision-makers are ensured by separating operational areas, we further argue that by installing a decision support system for single, closed operational areas or jurisdictions, computer assistance is more consistent, penetrative and thus more effective. This is particularly important for situations when single organizations "are assigned their own operational area, which is then to be operated independently such that the organization acts as an EOC" (cit. THW, translated).

In order to identify requirements for the design and the solution of such a decision support model we use two sources: first, in order to account for the experience of practitioners, we interviewed associates of the German Federal Agency for Technical Relief (THW), who were in direct contact with the first German search and rescue teams after the major earthquakes in Japan in March 2011 and who were knowledgeable with respect to on-site coordination. 
Second, we use knowledge and experience of scholars with domain expertise (literature review). As a result, we derive the requirements shown in Table 1.

\begin{tabular}{|c|c|c|}
\hline No. & Requirement & Motivation \\
\hline & \multicolumn{2}{|r|}{ Decision support model } \\
\hline 1 & $\begin{array}{l}\text { Classification of rescue } \\
\text { units and incidents } \\
\text { (German THW, } \\
\text { Wex et al. 2011) }\end{array}$ & $\begin{array}{l}\text { - Rescue units are heterogeneous in their skills. } \\
\text { - Incidents are heterogeneous in their needs. } \\
\text { - Heterogeneity affects assignment of rescue units to } \\
\text { incidents. }\end{array}$ \\
\hline 2 & $\begin{array}{l}\text { Non-Preemptiveness } \\
\text { (German THW) }\end{array}$ & $\begin{array}{l}\text { - In chaotic situations the extent and the level of } \\
\text { severity of incidents can be estimated only vaguely. } \\
\text { - It seems irresponsible to stop processing the } \\
\text { respective incident although further attention is } \\
\text { necessary and possible. }\end{array}$ \\
\hline 3 & $\begin{array}{l}\text { Incompleteness and } \\
\text { linguistic uncertainty of } \\
\text { information } \\
\text { (Fiedrich et al. 2000, } \\
\text { Rolland et al. 2010, } \\
\text { Comes et al. 2010) }\end{array}$ & $\begin{array}{l}\text { EOCs often face uncertain, unconfirmed, and } \\
\text { contradictory information } \\
\text { - Information is often described and assessed } \\
\text { subjectively by humans, thus linguistic estimations } \\
\text { are common. } \\
\text { - Uncertainty of information is not statistical in nature. }\end{array}$ \\
\hline & \multicolumn{2}{|r|}{ Decision support methodology } \\
\hline 4 & $\begin{array}{l}\text { Timeliness/efficiency } \\
\text { (Engelmann \& Fiedrich, } \\
\text { 2007; Reijers et al., 2007) }\end{array}$ & $\begin{array}{l}\text { - The critical deadline (first } 72 \text { hours after the } \\
\text { catastrophe) is essential for surviving } \\
\text { - Solution approaches must be efficiently applicable to } \\
\text { scenarios of realistic size. }\end{array}$ \\
\hline 5 & $\begin{array}{l}\text { Measurable effectiveness } \\
\text { (Sharda et al., 1988) }\end{array}$ & $\begin{array}{l}\text { Appropriateness of a decision support system and } \\
\text { methodology depends on the quality of the suggested } \\
\text { solution(s). } \\
\text { - Quality can be assessed (and measured) in terms of } \\
\text { how close the solution(s) come to the theoretical } \\
\text { optimum or to what extent the harm indicated through } \\
\text { state-of-the-art solutions are improved. } \\
\text { - Measurement of effectiveness is important for } \\
\text { assessing the appropriateness and improving the } \\
\text { quality of a decision support system methodology. }\end{array}$ \\
\hline
\end{tabular}

Table 1. Requirements for the decision support model and for decision support methodology

\section{$\underline{\text { Requirement 1: Classification of rescue units and incidents }}$}

The issue of allocating and scheduling rescue units during emergency response has been addressed only rarely in the literature. Fiedrich et al., (2000), Rolland et al. (2010), and Wex et al. (2011) all attest that rescue units' assignments and schedules are an understudied, yet highly relevant topic for IS research, and they suggest applying decision optimization models in a centralized manner, with a particular focus on the allocation of distributed rescue units to incidents. However, Rolland et al. (2010) neglect the fact that rescue units are diverse in their skills. Fiedrich et al. (2000) consider only one type of incident: earthquakes. Wex et al. (2011) 
take heterogeneous rescue units into account for coordination in a centralized way and they do not concentrate on one distinct disaster type only.

In the interview with a representative of the German THW, it was said that "[...] when several, differently-skilled rescue teams collaborate, it is often hard to strictly classify their structure, capabilities, and their behavior. In fact, rescue units are diverse in their capabilities and sizes. [...] Generally, incidents are classified into types, such that distinct specialized rescue units are required, although it is more than challenging to prioritize a scene and to tell when search-and-rescue or firefighting brigades need to be demanded." (cit. THW, translated) Accounting for this insight of practitioners, we argue that decision support systems need to consider heterogeneous types of incidents and distinct capabilities of rescue units. For example, units can be paramedics, fire brigades, or policemen. In cases where no detailed information is available, it seems straightforward to classify incidents coarse-grained and to assign one of the rescue units that is deemed most appropriate for addressing the incident. In other cases, more detailed information on incidents is available and can be matched with specific capabilities of rescue units.

\section{Requirement 2: Non-preemptiveness}

Once an incident has started to being processed, the processing rescue unit has, in principle, the option to stop its operation (preemption) and move to another location when a new, possibly much more severe incident needs attention (German THW). However, one can also argue that in chaotic situations where the extent and the level of severity of incidents can usually only be estimated vaguely, it seems irresponsible and also difficult to explain to affected persons to stop processing the respective incident although further attention is necessary and possible. Under these complex circumstances which are often found in emergency response practice, this approach has been affirmed by the German THW for some cases.

\section{$\underline{\text { Requirement 3: Incompleteness and linguistic uncertainty of information }}$}

During any large-scale natural disaster much information remains unavailable or uncertain (Fiedrich et al., 2000) and "[...] decision support systems used in disaster management must cope with the complexity and uncertainty involved with the scheduling assignment of differentially-skilled personnel and assets to specific tasks.” (Rolland et al., 2010). Thus, commanders of EOCs often face uncertain, unconfirmed, and even contradictory information (Comes et al., 2010). While information on available rescue units and their capabilities is usually certain, information on incidents, including the level of severity, processing times and travel times, is usually not. As this information is often described and assessed by humans, linguistic estimations are common. Thus, we argue that decision support systems need to account for linguistic, non-probabilistic informational uncertainty.

However, in the literature there is a lack of how informational uncertainty due to linguistic assessments can be handled in emergency response situations. In the autonomous agents community, several works have been proposed that handle task allocation in uncertain environments mainly by using auctions. But they either do not explicitly coordinate rescue agents or they do not fully consider the characteristics of the emergency response domain (Nair et al., 2002; Ramchurn et al., 2008).

Recalling that uncertainty in chaotic emergency situations occurs due to incomplete and imprecisely stated information and not due to statistical uncertainty, we do not suggest a probabilistic optimization model but a decision model that draws on fuzzy set theory, fuzzy arithmetic, and fuzzy optimization. 


\section{Requirement 4: Timeliness/efficiency}

The first 72 hours after any catastrophe, the so-called critical deadline, are essential for surviving (Engelmann \& Fiedrich, 2007; Reijers et al., 2007). Therefore, any research presenting quantitative artifacts must demonstrate its ability to (re-)act timely in real-world applications. As a consequence, any decision support system has to provide allocation and scheduling suggestions that are not only practically feasible and justifiable (in terms of specific criteria to be defined) but that are also made speedily available to aid organizations. As a consequence, solution approaches must be efficiently applicable to scenarios of realistic size.

\section{$\underline{\text { Requirement 5: Measurable effectiveness }}$}

The appropriateness of a decision support system and the embedded methodology depends on the quality of the suggested solution(s) (Sharda et al., 1988). This quality can be assessed in terms of how close the solution(s) come to the theoretical optimum or to what extent the harm indicated through state-of-the-art solutions are improved based on expert opinions. While the former requires knowing the theoretical optimum, which is computationally expensive even for medium-size instances, the latter requires benchmarks with best practice solutions. In both cases the effectiveness can be measured, which is an important requirement for assessing the appropriateness and improving the quality of a decision support system methodology.

\section{A Fuzzy Decision Support Model}

In this section, we suggest a non-linear fuzzy decision support model. We first briefly introduce into the key concepts of fuzzy set theory and fuzzy optimization; for a comprehensive overview of these areas, see the works of Buckley \& Eslami (2002) and Klir \&Yuan (1995). Then we provide an overall problem description before we relate our problem to similar problems discussed in the optimization literature. Finally, we present our mathematical model and analyze its complexity.

\section{Fuzzy Set Theory}

Fuzzy set theory generalizes traditional set theory by providing for a degree of membership that indicates if an element belongs to a fuzzy set, in contrast to (crisp) set theory, wherein an element explicitly either comes with a set or not. A specific type of a fuzzy set is a fuzzy number (Buckley \& Eslami, 2002), which is formally defined by $\left\{\left(x, \mu_{\widetilde{N}}(x)\right) \mid x \in R\right\}, \mu_{\widetilde{N}}: R \rightarrow$ $[0,1]$, where $\widetilde{N}$ is referred to as fuzzy number. $\mu_{\widetilde{N}}$ is denoted as the membership function of $\widetilde{N}$, and it outputs the degree with which $x \in R$ belongs to $\widetilde{N}$. For example, the fuzzy number $\widetilde{10}$ which is to be equivalently seen as "real numbers close to ten" may be given by the membership function $\mu_{\widetilde{10}}(x)=\left(1+(x-10)^{-2}\right)^{-1}\left(\mathrm{x} \in R^{\neq 10}\right), \mu_{\widetilde{10}}(10)=1$. Note that the membership function differs from a probability density function in two regards: $\int_{-\infty}^{\infty} \mu_{\widetilde{N}}(x) d x$ does not need to equal 1 , and it mirrors the subjective attitude of an individual rather than reflecting statistical evidence. This is advantageous in cases where probabilities or exact data is not available, but subjective estimates of experienced experts are given. In the emergency response setting such cases are typically prevalent. The Fuzzy Decision Model makes use of the concept of symmetric triangular fuzzy numbers. A triangular fuzzy number $\mathrm{N}=(\mathrm{a}, \mathrm{b}, \mathrm{c})$, $\mathrm{a}<\mathrm{b}<\mathrm{c},\{\mathrm{a}, \mathrm{b}, \mathrm{c}\} \in \mathrm{R}$, is a fuzzy set over $\mathrm{R}$, with the membership function 


$$
\mu_{N}(x)=\left\{\begin{array}{c}
\frac{x-a}{b-a}, \text { if } a \leq x<b \\
\frac{c-x}{c-b}, \text { if } b \leq x \leq c \\
0, \text { otherwise }
\end{array}\right.
$$

If $l:=(c-b)=(b-a)$, then the triangular fuzzy number is symmetric. We use symmetric fuzzy numbers with $l=0.1 * \mathrm{~b}$ depending on the degree of uncertainty we are facing. This corresponds to "10\% fuzziness". However, while for many crisp optimization problems algorithms are available, this is not true for fuzzy optimization problems (Buckley \& Jowers, 2008). Thus, we apply a Monte Carlo simulation for the computational evaluation in the follow-up.

\section{Problem Description}

The model is designed to schedule and assign various rescue units to incidents. It favors commanders with decision autonomy by delivering allocation solutions and schedules for all rescue units employed. The evolving question is how these units can be scheduled and assigned to incidents such that the sum of all completion times, which are individually multiplied by the individual factors of destruction, can be minimized. Factors of destruction indicate the (ordinal) levels of severity of incidents. We refer to this problem as the Rescue Unit Assignment and Scheduling Problem (RUASP).

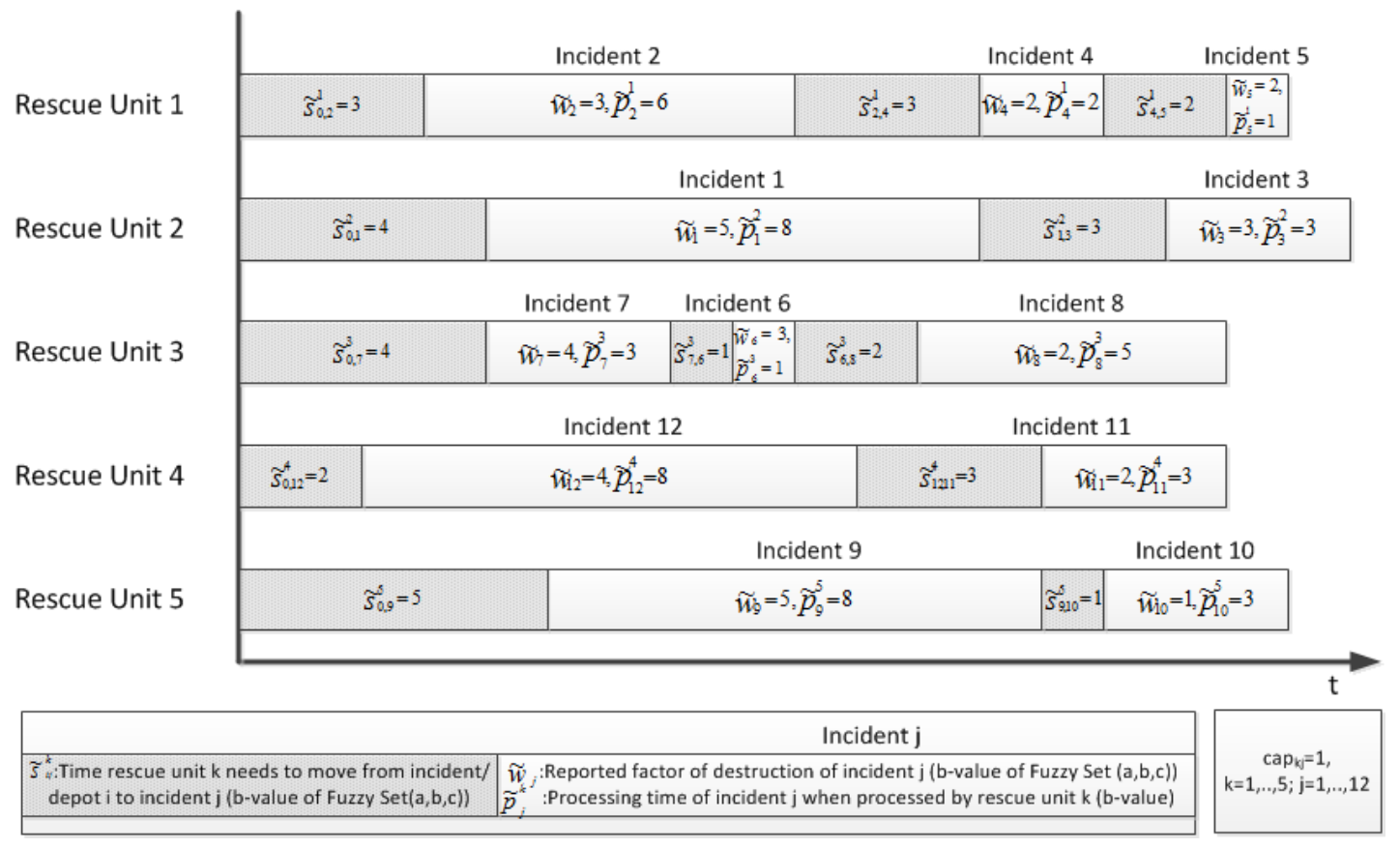

Figure 1. Desideratum: optimal schedules and assignments.

We consider a situation in which the number of available rescue units is lower than the number of incidents that need to be processed. This ratio accounts for a typical natural disaster situation: "During any large-scale disaster, there tend to be more incidents than rescue units. This is especially true within those critical minutes of the chaos phase." (cit. THW, translated) An incident can be processed by a rescue unit only if this rescue unit features the specific capability that is required to process this incident. Two types of time spans are relevant: a) travel times that rescue units need to travel between two incident locations, b) processing times. We illustrate the RUASP description in Figure 1, which shows a feasible and valid solution of a RUASP instance with 5 rescue units and 12 incidents. In this instance, the vague level of severity (factor of destruction $\widetilde{W_{J}}$ ) of incidents varies between 1 
and 5. The sample schedule accounts for the specific requirements of the incidents as each rescue unit $\mathrm{k}$ features the respective capability that is required by incident $\mathrm{j}\left(\mathrm{cap}_{\mathrm{kj}}=1\right)$.

\section{Relationship to Routing and Scheduling Problems}

We now relate (the crisp version of) our problem to decision problems in the optimization literature.

Our problem is related to the multiple Travelling Salesman problem (mTSP), which is a generalization of the TSP and a relaxation of the Vehicle Routing Problem (VRP), with the capacity restrictions removed (Bektas, 2006). Mapping rescue units to salesmen and incidents to cities/nodes, and requiring that rescue units need to return to a central depot as fictitious incident with severity level 0 , we can model capabilities by setting corresponding decision variables of the mTSP to 0 . Preemptiveness is inherently included in the mTSP. However, while we can aggregate processing times and travel times in the RUASP to overall travel times, it remains the problem that travel times in the mTSP are not salesman-specific. This property can be modeled through providing for salesmen-specific travel times between two cities, thus leading to the problem "mTSP with salesman-specific travel times". We can thereby also model that rescue units start at different depots. The way this modification changes the mTSP depends on the particular mTSP problem specification. In their mTSP review paper, Bektas (2006) present four different specifications. Among these specifications, only the flow based formulation can be accordingly modified straightforward as it is the only specification that uses three-index decision variables (for two cities and one salesman). Drawing on this specification, the mTSP can be easily extended to the mTSP with different travel times by leaving all constraints unchanged and substituting only the objective coefficients $\mathrm{c}_{\mathrm{ij}}$ by $\mathrm{c}_{\mathrm{ij}}{ }^{\mathrm{k}}$, with $\mathrm{k}$ being the index of the salesman and $\mathrm{i} ; \mathrm{j}$ being the index of the city. Finally, a serious issue is the consideration of the objective to minimize the sum of weighted completion times. In contrast, in the mTSP the objective value depends only on the edges that are travelled but not on the order in which they are travelled. The latter property is inherently included in the RUASP. Considering this property leads to a problem that we denote as "mTSP with salesman-specific travel times under minimizing the sum of weighted visiting times". We are not aware of any paper that addresses a problem of this structure. The VRP shares this issue of the mTSP, and we are not aware of any VRP extension that allows for modeling our problem. To sum up, the RUASP is related to both the mTSP and the more general VRP but it is neither a specialization nor a relaxation of any of these problems. Consequently, neither an exact mTSP algorithm nor an exact VRP algorithm can be regarded as an exact RUASP algorithm.

The RUASP is also related to problems in the scheduling literature. If we map rescue units to machines, incidents to jobs and travel times to setup times, then the RUASP is similar to the "parallel-machine scheduling problem with unrelated machines, non-batch sequencedependent setup times, and a weighted sum of completion times as the objective", classified as $\mathrm{R} / \mathrm{ST}_{\mathrm{SD}} / \sum \mathrm{w}_{\mathrm{j}} \mathrm{C}_{\mathrm{j}}$ in the scheduling literature (Allahverdi et al., 2008). The RUASP generalizes this scheduling problem, as the former provides for machine specific setup times between two jobs while in the latter setup times depend only on the jobs, i.e. the RUASP becomes an $\mathrm{R} / \mathrm{ST}_{\mathrm{SD}} / \sum \mathrm{w}_{\mathrm{j}} \mathrm{C}_{\mathrm{j}}$ scheduling problem if setup times are machine-independent. Capabilities of the RUASP can be modeled by setting the corresponding decision variables to 0 . With regard to the problem formulation of RUASP, any formulation of the scheduling problem $\mathrm{R} / \mathrm{ST}_{\mathrm{SD}} / \sum \mathrm{w}_{\mathrm{j}} \mathrm{C}_{\mathrm{j}}$ may be used and modified so that the property that different rescue units need different travel times between the locations of the incidents is accounted for. However, according to the review paper by Allahverdi et al. (2008), there is only one paper on this scheduling problem (Weng et al., 2001). While this paper suggests a recursive objective 
function, it specifies the constraints at high level only. Thus, their model formulation is too generic for our intention to suggest an optimization model.

\section{Mathematical Model}

We define completion times as the sum of processing times and the time the incident had to "wait" until being processed by a qualified rescue unit. This "waiting time" consists not only of processing times of incidents that have been processed previously by the assigned unit but also of the time needed to move from one incident to be processed to the next.

In the assumed setting, we propose that a) the multiplication of completion times and factors of destruction $\widetilde{w_{J}}$ is an adequate proxy for the quality of emergency response, $b$ ) each incident can be processed by at most one unit at a time with each unit processing at most one incident at a time, c) processing is non-preemptive, and d) some data (processing times $\widetilde{p_{l}^{k}}$, severity of incidents $\widetilde{w_{J}}$, and travel times $\widetilde{s_{l J}^{k}}$ ) is available, deterministic, but highly uncertain and therefore not crisp. A discussion of these assumptions is included in our conclusions. Summarizing the restrictions and requirements from above, this decision model can be formulated as a non-linear binary optimization model. The mathematical formulation is provided below:

$$
\begin{aligned}
& \min \sum_{j=1}^{n} \widetilde{w_{J}}\left(\sum_{i=0}^{n} \sum_{k=1}^{m}\left[\widetilde{p_{l}^{k}} Y_{i j}^{k}+\left(\widetilde{p_{J}^{k}}+\widetilde{s_{l j}^{k}}\right) X_{i j}^{k}+Y_{i j}^{k}\left(\sum_{l=0}^{n} X_{l i}^{k} \widetilde{s_{l l}^{k}}\right)\right]\right) \\
& \sum_{i=0}^{n} \sum_{k=1}^{m} X_{i j}^{k}=1, \quad j=1, \ldots, n \\
& \sum_{j=1}^{n+1} \sum_{k=1}^{m} X_{i j}^{k}=1, \quad i=1, \ldots, n \\
& \sum_{j=1}^{n+1} X_{0 j}^{k}=1, \quad k=1, \ldots, m \\
& \sum_{i=0}^{n} X_{i(n+1)}^{k}=1, \quad k=1, \ldots, m \\
& Y_{i l}^{k}+Y_{l j}^{k}-1 \leq Y_{i j}^{k} \text {, } \\
& i=0, \ldots, n ; j=1, \ldots, n+1 ; k \\
& =1, \ldots, m ; l=1, \ldots, n \\
& \sum_{i=0}^{n} X_{i l}^{k}=\sum_{j=1}^{n+1} X_{l j}^{k}, \quad l=1, \ldots, n ; k=1, \ldots, m \\
& X_{i j}^{k} \leq Y_{i j}^{k}, \quad i=0, \ldots, n ; j=1, \ldots, n+1 ; k \\
& =1, \ldots, m \\
& Y_{i i}^{k}=0, \quad i=0, \ldots, n+1 ; k=1, \ldots, m \\
& \sum_{j=1}^{n+1} X_{i j}^{k} \leq \operatorname{cap}_{k j}, \quad i=1, \ldots, n ; k=1, \ldots, m
\end{aligned}
$$




$$
\begin{aligned}
& X_{i j}^{k}, Y_{i j}^{k} \in\{0,1\}, \\
& \quad i=0, \ldots, n ; j=1, \ldots, n+1 ; k \\
& =1, \ldots, m
\end{aligned}
$$

In addition to the real incidents $1, \ldots, \mathrm{n}$ we need to add two fictitious incidents ' 0 ' and ' $\mathrm{n}+1$ ' with $\widetilde{p_{0}^{k}}=\widetilde{p_{n+1}^{k}}=0$, and $\widetilde{s_{0}^{k}}$ to be the estimated time that agent $\mathrm{k}$ needs to move from its starting location (defined as incident $\mathrm{i}=0$ ) to the location of incident $\mathrm{j}$, and $s_{J(n+1)}^{\bar{k}}=0$ for all rescue units $\mathrm{k}$. The objective function $(\mathrm{O})$ of the model minimizes the total weighted completion times over all incidents. Two decision variables $X_{i j}^{k}$ and $Y_{i j}^{k}$ are introduced indicating a mediate or immediate predecessor relationship between $\mathrm{i}$ and $\mathrm{j}$ when processed by rescue unit $\mathrm{k} . \widetilde{w_{j}}$ is the reported factor of destruction of incident $\mathrm{j}$ and is modeled as a triangular fuzzy number. Consequently, the lower the factor of destruction, the less severe is the incident. An explanation of the other mathematical terms used is provided in Table 2.

\begin{tabular}{|c|l|}
\hline Decision Variable & \multicolumn{1}{|c|}{ Interpretation } \\
\hline $\mathrm{X}_{\mathrm{ij}}^{\mathrm{k}}$ & $\begin{array}{l}\mathrm{X}_{\mathrm{ij}}^{\mathrm{k}}=1 \text { if incident } \mathrm{i} \text { is processed immediately before } \\
\text { incident } \mathrm{j} \text { by rescue agent } \mathrm{k}, \text { and } 0 \text { otherwise }\end{array}$ \\
\hline $\mathrm{Y}_{\mathrm{ij}}^{\mathrm{k}}$ & $\begin{array}{l}\mathrm{Y}_{\mathrm{ij}}^{\mathrm{k}}=1 \text { if incident } \mathrm{i} \text { is processed before incident } \mathrm{j} \text { by } \\
\text { rescue agent } \mathrm{k}, 0 \text { otherwise }\end{array}$ \\
\hline Fuzzy Parameters & \multicolumn{1}{c|}{ Interpretation } \\
\hline$\widetilde{\mathrm{p}_{\mathrm{l}}^{\mathrm{k}}}$ & $\begin{array}{l}\text { Processing time that agent } \mathrm{k} \text { needs to process incident } \mathrm{i}, \\
\mathrm{p}_{\mathrm{l}}^{\mathrm{k}}=\infty \text { if agent } \mathrm{k} \text { is incapable of processing incident } \mathrm{i}\end{array}$ \\
\hline$\widetilde{\mathrm{s}_{\mathrm{j}}^{\mathrm{k}}}$ & $\begin{array}{l}\text { Travel time that agent } \mathrm{k} \text { needs to move from location of } \\
\text { incident } \mathrm{i} \text { to location of incident } \mathrm{j}\end{array}$ \\
\hline$\widetilde{\mathrm{w}_{\mathrm{j}}}$ & $\begin{array}{l}\text { Reported factor of destruction of incident } \mathrm{j} \text { equivalent } \\
\text { to the severity level of an incident }\end{array}$ \\
\hline Crisp Parameter & \multicolumn{1}{c}{ Interpretation } \\
\hline cap ${ }_{\mathrm{kj}}$ & $\begin{array}{l}\text { cap } \\
\text { incident } \mathrm{i} \text {, and } 0 \text { otherwise }\end{array}$ \\
\hline
\end{tabular}

\section{Table 2. Explanation of mathematical terms}

Constraint $(\mathrm{C} 1)$ ensures that for each real incident there is exactly one incident that is processed immediately before. Similarly, (C2) ensures that for each real incident there is exactly one incident that is processed immediately thereafter. Constraints $(\mathrm{C} 3)-(\mathrm{C} 4)$ guarantee that in a feasible solution each rescue agent starts processing the fictitious incident 0 and ends processing the fictitious incident $\mathrm{n}+1$, respectively. (C5) accounts for the transitivity criterion of any predecessor relationship. Yet, if an immediate predecessor for a specific incident ' 1 ' exists, there also has to be a successor (C6). (C7) indicates that an immediate predecessor is a general predecessor. (C8) prohibits a reflexive, direct or indirect predecessor relationship. (C9) ensures that a rescue unit that is assigned to an incident possesses the required, incident- 
specific capability. (C10) makes the model a binary program. (C11) declares if a rescue unit is capable of operating an incident or not. (C12) defines all other parameters used. Each feasible solution of the minimization model represents a valid schedule and assignment for all units.

This Fuzzy Decision Model is especially able to manage informational overload and linguistic uncertainty by integrating fuzzy parameters (Requirement 4): impreciseness in reports from on-site forces is prevalent when determining travel and processing times, as well as the severity of incidents. Furthermore, the model is apt to assist (decentralized) commanders with decision autonomy but does not require exact information about all parameters used. In the adjacent sections, it will be shown that the model is also adequate to deliver timely results within decent runtimes when applying the solution heuristic (Requirement 1).

The idea to search for something optimal during any disaster is questionable and can be doubted, especially when integrating uncertain information (fuzzy parameters) into the model. We therefore talk about the quest for the most effective allocations of rescue units in an uncertain setting. Disaster situations are evolving very fast sometimes (based on incoming information about the situation, incoming new resources, or on status changes of existing resources). Even though the presented approach seems to not account for this inherent dynamic and to be static, we explicitly suggest running the optimization of weighted completion times anew once other incidents appear or rescue units become idle (continuous optimization process). This way, alternatives and decisions can also be revisited and alterations can be integrated.

\section{Complexity of the problem}

As the subsection "Relationship to routing and scheduling problems" shows, the crisp version of the RUASP is a generalization of the machine scheduling problem "Identical parallel machine non-preemptive scheduling with minimization of sum of completion times". We show in the Appendix that both the crisp version of the RUASP and the fuzzy version are NPhard and thus computationally inefficient. As we face instances in practice, that need to be solved in near-time, we suggest a Monte Carlo simulation as heuristic method. In the absence of knowledge of optimal solutions, we do not know lower bounds for the minimization instances, but we know solutions that would result from applying a greedy heuristic. Recapitulating the greedy approach, we assume that the most severe incident is assigned to the closest, idle rescue unit. The evaluation of all Monte Carlo results is based on the comparison with this benchmark indicating the proportionate reduction of harm. Implementations were written in the numerical computing environment MATLAB.

\section{Monte Carlo Heuristic}

As the RUASP is a computationally hard problem we suggest a Monte Carlo heuristic for the RUASP. The decision to select a Monte Carlo approach is based on the following reasons: (1) The complexity of the RUASP is high due to the many constraints, and we assume that the number of local optima is high so that local search procedures would easily lead to "bad" local optima. In more complex scenarios, “[...] evaluation procedures rely a great deal on trial and error.” (Buxey, 1979; p. 566) In contrast, a Monte Carlo algorithm overcomes this shortcoming and its runtime is scalable through the number of applied iterations. (2) Monte Carlo simulation is flexible with regard to future extensions of the optimization model, such as the co-allocation of rescue units.

The key idea of generating a feasible solution in our Monte Carlo simulation is that incidents are iteratively scheduled in two stages: in stage one, an incident is assigned randomly to one 
of the $\mathrm{D} \%$ most appropriate rescue units, where appropriateness is defined based on processing times. The motivation of this procedure is based on avoiding both a) assignments of incidents to units that require an extremely long time for processing (thus, a parameter D in $[0 ; 100]$ is used), and b) myopic assignments of incidents to units that require the shortest processing time among all units (thus, randomness is included). In stage two, the incident is inserted into the incident queue of the previously selected rescue unit. The criterion for determining the position of the new incident in the queue is based on a weighted ratio of the severity of incident $\mathrm{w}$ and the time $\mathrm{p}$ it takes the selected rescue unit to process this incident. Each queue lists its incidents in descending order of (w/p)-values. In more detail, the heuristic proceeds as follows (cmp. the Pseudo-code notation in the Appendix):

The Monte Carlo heuristic requires two input parameters: $\mathrm{D}$ in $[0 ; 100]$ is used for the selection of rescue units (see step 10), ITERATIONS is the number of feasible solutions generated; we set $\mathrm{D}=90$ and ITERATIONS $=1,000$ based on pretesting results. As initialization, the currently best solution value is set to infinity and the currently best solution is set to undefined (step 1), the current number of iterations is set to 0 (step 2), the cumulated processing times are set to 0 for each rescue unit (step 3), the current incident queues are set to empty for each rescue unit (step 4), and we define $I^{*}$ as the set of currently unassigned incidents (step 5). The incidents are now processed iteratively (steps 6-16): We define $\mathrm{K}^{*}$ as the set of all rescue units that are capable of processing incident (step 7) and rearrange $\mathrm{K}^{*}$ in ascending order of cumulative processing times (step 9). If there is no rescue unit that has the capability to process the incident, the algorithm terminates unsuccessfully (steps 8 and 21). The algorithm now randomly selects a rescue unit with one of the $\mathrm{D} \%$ lowest cumulative processing times (step 10). The purpose of introducing this element of randomization is the avoidance of greedy assignments of rescue units to the incident while contemporaneously avoiding assignments of rescue units with extremely high cumulative processing times. The cumulative processing time of the selected unit is then updated (step 11), which concludes stage 1 . In stage 2, the incident is inserted into the queue of unit queue(unit) such that the queue is ordered in ascending order of values (fact_destruct(i)/processing time(unit, i)), with i being the position of the incident in the queue (steps 12-14), and the incident is removed from the set of incidents that still need to be assigned (step 15). If all incidents have been assigned (step 8), then the current schedule is compared with the best known schedule, which is contingently updated (step 17). The algorithm terminates successfully if ITERATIONS feasible solutions have been generated (steps 18-20).

\section{Computational Evaluation}

We evaluate the suggested Monte Carlo heuristic through computational experiments that were implemented in the numerical computing environment MATLAB. We first describe how we generate scenarios before we present the results. This presentation benchmarks the Monte Carlo solutions with solutions that would have resulted from the best practice approach described above, which we refer to as the "greedy approach".

\section{Data Generation}

The generation of data for RUASP instances is based on the answers of the German THW interviewee and on suggestions of the literature. We assume that processing times substantially exceed travel times between incidents' locations, due to the hypothesis that urban areas are endangered more often than rural areas, which results in high density of incident locations. The factors of destruction indicate levels of severity and express five different stages for each incident. We use the advisory system concerning threat conditions and risks introduced by the U.S. Department of Homeland Security, which provides for the following stages: low (1), guarded (2), elevated (3), high (4), and severe (5) harm. The 
description of these stages is linguistic, which demonstrates that the severity of threat conditions is assessed vaguely in practice. In our context, incidents with "little damage" or "minor injuries" may be classified as "low", while incidents with "collapsed buildings and trapped people" may be classified as "severe". All data related to processing and travel times and to the level of severity are modeled with (symmetric triangular) fuzzy numbers. These numbers are generated in two steps: in step one, the "center" b of the fuzzy number $\widetilde{N}$ $\left(\mu_{\widetilde{N}}(b)=1\right)$ is generated following the distributions shown in Table 3. In step 2, the respective symmetric, triangular fuzzy number is determined by $\widetilde{N}=(0.9 * \mathrm{~b}, \mathrm{~b}, 1.1 * \mathrm{~b})$. Based on the description of emergency operations by New South Wales Government (n.d.), we assume that five types of rescue units with different capabilities are available (e.g., paramedics, fire brigades, police enforcement, military forces, or volunteers with various other skills).

Our simulation includes the generation of instances of different size in terms of the numbers of incidents and rescue units. We assume that no more than 20 rescue units are available and 200 incidents need to be processed in one instance as commanders operate within their own operational area only. We discuss the motivation for and implication of this assumption in the discussion section in more detail.

\begin{tabular}{|c|c|c|}
\hline Parameter & $\begin{array}{l}\text { Value, Range, } \\
\text { Distribution }\end{array}$ & Rationale \\
\hline Rescue units & $\{10,20\}$ & \multirow{2}{*}{$\begin{array}{l}\text { Realistic numbers of rescue units and } \\
\text { incidents within operational areas }\end{array}$} \\
\hline Incidents & $\{20,50,100,200\}$ & \\
\hline $\begin{array}{l}\text { Processing } \\
\text { times } \widetilde{p_{k l}}\end{array}$ & $\begin{array}{l}\text { Normally distributed: } \\
\mu=20, \sigma=10\end{array}$ & \multirow{2}{*}{$\begin{array}{l}\text { Occurrence of disasters close to } \\
\text { overcrowded areas (thus: low travel times } \\
\text { between incidents); WLOG: significant } \\
\text { endurance of (mean) processing times to } \\
\text { (mean) travel times (factor: 20:1) }\end{array}$} \\
\hline $\begin{array}{l}\text { Travel times } \\
\widetilde{S_{k l}}\end{array}$ & $\begin{array}{l}\text { Normally distributed: } \\
\mu=1, \sigma=0.3\end{array}$ & \\
\hline $\begin{array}{l}\text { Factors of } \\
\text { destruction } \widetilde{W_{l}}\end{array}$ & $\begin{array}{l}\text { Random Integer: } \\
\{1, . ., 5\}\end{array}$ & $\begin{array}{l}\text { Distinct risk levels introduced by the U.S. } \\
\text { Department of Homeland Security }\end{array}$ \\
\hline $\begin{array}{l}\text { Capabilities } \\
\left\{A_{1}, \ldots, A_{n}\right\}\end{array}$ & $\begin{array}{l}\mathrm{n}=5 \\
\text { A1: Search and Rescue } \\
\text { A2: Paramedics / } \\
\text { Medical Retrieval } \\
\text { A3: Fire Brigades } \\
\text { A4: Police Units } \\
\text { A5: Special Casualty } \\
\text { Access Team }\end{array}$ & $\begin{array}{l}\text { Distinction of units' types and skills } \\
\text { extending the classification of (New } \\
\text { South Wales Government) }\end{array}$ \\
\hline Iterations & 1,000 & $\begin{array}{l}\text { No significant improvements in the } \\
\text { objective value beyond this point }\end{array}$ \\
\hline
\end{tabular}

Table 3. Settings in randomly generated scenarios. 


\section{Results}

We benchmark all Monte Carlo simulation results with the results generated by the greedy policy that represents current best practice (the most severe incident is assigned the closest, idle rescue unit and the remaining idle rescue units are allocated to incidents in the same manner). We present proportions of Monte Carlo simulation results to those of the greedy policy by means of box plots. Each value represents the ratio of objective values (total weighted completion times) between the Monte Carlo simulation and the greedy heuristic. The box plots comprise the means (red dash), the quartiles (ends of box), the lowest/highest datum within 1.5 IQR (whiskers), and all outliers (stars). Thus, if both the Monte Carlo simulation and the benchmark provide the same assignment and schedule, and thus the same objective value, the ratio would be presented as ' 1.0 '. If the Monte Carlo heuristic performs superior to the benchmark, i.e. the total weighted completion time is lower, the ratio is below 1.0 .

7 different scenarios (with 10 instances each) have been generated randomly according to the preconditions in Table 3. All Monte Carlo simulations have been aborted after 1,000 iterations to allow for acceptable runtimes in practice. No significant improvements of the results have been identified thereafter. As 1,000 iterations of the Monte Carlo simulation were run within minutes on a standard PC, the procedure turns out to be efficient for being applied in practice.

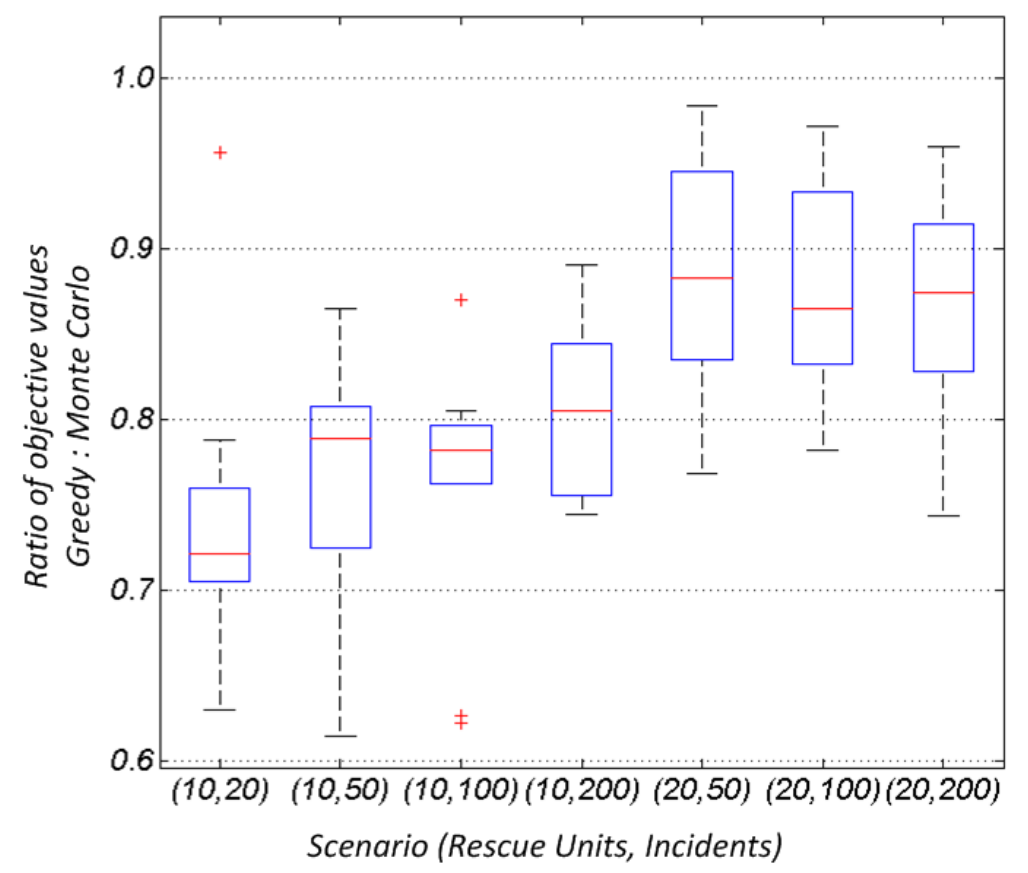

Figure 2. Results indicating the ratio between the heuristics used.

As Figure 2 indicates, the Monte Carlo simulation performs better than the greedy policy. Ranges of deviation of simulation results are acceptable for all problem scenarios and none of the results exceeds the benchmark value (proportions $\leq 1.0)$. In scenario $(10,20)$, the Monte Carlo simulation is even able to generate a total weighted completion time of less than a quarter of which would have been caused by the greedy heuristic. The Monte Carlo heuristic allows for damage reductions of at least $10 \%-20 \%$ on average compared to the benchmark. Apparently, the ratios are closer to $100 \%$ the more complex the scenarios get (starting from 20 rescue units). This phenomenon is not surprising as the fraction of the solution space that gets evaluated by the Monte Carlo simulation declines with increasing instance size. A countermeasure would be to increase the number of iterations in the Monte Carlo simulation, which in turn would require having available more computing power than we had. Based on the results at hand, we observe a high coefficient of variation for some scenarios, which we 
explain as a consequence of "fuzzifying" the parameters, which in turn may reflect the cost of incorporating linguistic vagueness.

All results were subjected to the Shapiro-Wilk test (Shapiro \& Wilk, 1965) to prove normality. Pre-proven normality holds as necessary condition for further analysis: results of significance tests expressed that the simulations of all our models do outperform the benchmark within the confidence intervals of a 95\% significance level except for instance $(10,100)$, where a normal distribution of the results was rejected. Our results attest that solving our models with Monte Carlo outperforms the heuristic, which is applied in practice.

\section{Discussion}

As the results show, the application of the Monte Carlo heuristic is superior to the greedy heuristic which we previously identified as best practice in accordance to our interviews with the German THW. Beyond improved effectiveness through reduced overall harm, we see the benefit of our formal approach not only in the algorithmic superiority of the suggested Monte Carlo heuristic but also in the formal decision model itself as it provides the basis for designing, implementing and applying algorithms. As stated in the interviews, in current practice the greedy policy is conducted manually so that in large instances even the solution quality of the greedy policy may not be achieved due to high complexity. These arguments call for the development and deployment of optimization algorithms and IT-based decision support systems.

At the same time it should be noticed that - accounting for the term "support" - decisions on actions in emergency response should not be automated but still made by humans who are domain experts and who may want to overrule suggestions of the decision support system based on situation-specific knowledge that is not modeled in the decision support system. Therefore, our claim to computationally optimize an emergency response setting may be relaxed due to the decision autonomy of the commanders.

Based on the inherent uncertainty in emergency response, the sharp definition of fuzzy set numbers in this study being symmetric and triangular may seem contradictory. Therefore, we designed our optimization model and the corresponding solution heuristics in such a way that they promote flexibility in regard to other settings of fuzzy numbers, e.g. trapezoidal or bellshaped fuzzy numbers.

An important issue in emergency response is the dynamics inherent in chaotic situations. These dynamics can manifest in new incidents, changed requirements of incidents, changes in the available resources and their capabilities, changes in traffic infrastructure etc. As a consequence, any decision support should account for these dynamics. Our heuristic (and any other solution approach) should thus be performed iteratively, with each iteration addressing a particular situation and planning horizon. If new information becomes available, a new situation occurs and a new iteration of the applied solution procedure may deem necessary. In this new situation, actions already been taken need to be considered; for example, incidents that have been (started being) processed should be removed (due to non-preemptiveness) and positions of rescue units need to be adapted etc., i.e. the extent to which the old plan has been implemented impacts the new plan. Following this path allows for accounting for dynamics in emergency response situations. A consequence of considering changed situations and applying solution algorithms iteratively is that the size of the instances can be assumed to be moderately large. Thus, we argue that limiting our instances to sizes of 200 incidents and 20 rescue units does not limit the applicability of our heuristic in practice and the significance of our results. 


\section{Conclusion}

The management of natural disasters poses immense challenges ranging from informational uncertainty to the problem of coordinating distributed, heterogeneous rescue units since disasters continue to hit our societies. Although NDM has evolved to a research discipline where IS artifacts have already been proposed, decision support procedures for assignments and schedules of rescue units have mostly been neglected in research.

Addressing this lack in research, the paper proposes a quantitative decision support model for the allocation of distributed, heterogeneous rescue units based on fuzzy set theory to deal with non-statistical informational uncertainty. Requirements identified in the literature and in interviews are accounted for. The suggested Monte Carlo solution heuristic offers decision support timely to any commander. While the proposed decision model may be particularly useful in the presence of complex situations with large numbers of rescue units and incidents, any assignments and schedules of rescue units determined through computation are not intended to replace the actual decision making process of commanders but may serve as valuable decision support only.

Due to the computational hardness of our decision model, we draw on Monte Carlo simulation and computationally demonstrated its benefits. The results show that there is large potential to improve a greedy heuristic to allocate and schedule rescue units. To conclude, we are aware that our research still has some limitations and invites for various streams of future work: (1) We exclude the possibility that rescue units may fatigue and thus refrain from a reduction in performance of rescue units over time. (2) Our model does not account for time windows of incidents. Such windows are appropriate when casualties have a finite "time to live" to be rescued. (3) The model does not consider pre-emptive approaches. (4) As real-life data-sets merely exist, all scenarios had to be randomly generated. Thus, empirical research is necessary to gather more realistic data. (5) Our model of capabilities and requirements can be extended in order to provide options for cooperation of rescue units. (6) As our problem is related to problems in the routing and scheduling literature, solution heuristics proposed in these domains may be adapted and tested for effectiveness.

\section{References}

Airy, G., Mullen, T., \& Yen, J. (2009). Market Based Adaptive Resource Allocation for Distributed Rescue Teams. In J. Landgren and S. Jul (Eds.), Proceedings of the 6th International Conference on Information Systems for Crisis Response and Management (ISCRAM). Gothenburg, Sweden, May 10-13, 2009.

Allahverdi, A., Ng, C., Cheng, T., \& Kovalyov, M. Y. (2008). A survey of scheduling problems with setup times or costs. European Journal of Operational Research, 187 (3), 985-1032.

Altay, N., \& Green III, W. G. (2006). OR/MS research in disaster operations management. European Journal of Operational Research, 175(1), 475-493.

Bektas, T. (2006). The multiple traveling salesman problem: an overview of formulations and solution procedures. Omega, 34 (3), 209-219.

Blazewicz, J., Dror, M., \& Weglarz, J. (1991). Mathematical programming formulations for machine scheduling: A survey. Operations Management Research, 51(3), 283-300.

Buckley, J. J., \& Eslami, E. (2002). An Introduction to Fuzzy Logic and Fuzzy Sets. Heidelberg, Germany: Physica Verlag.

Buckley, J. J., \& Jowers, L. J. (2008). Monte Carlo Methods in Fuzzy Optimization. Berlin, Heidelberg, New York: Springer-Verlag. 
Chawla, S. (2011). Japan Earthquake Resources: Aid Organizations, Charities and the Travel Industry Response. Retrieved September 25, 2012, from

http://www.petergreenberg.com/b/Japan-Earthquake-Resources:-Aid-Organizations,Charities-and-the-Travel-Industry-Response/-651186966021527775.html.

Chen, R., Sharman, R., Rao, H. R., \& Upadhyaya, S. J. (2008). Coordination in emergency response management. Communications of the ACM, 51(5), 66-73.

Comes, T., Conrado, C., Hiete, M., Kamermans, M., Pavlin, G., \& Wijngaards, N. (2010). An intelligent decision support system for decision making under uncertainty in distributed reasoning frameworks. In S. French, T. Tomaszewski, \& C. Zobel (Eds.), Proceedings of the 7th International ISCRAM Conference. Seattle, USA, May 2-5, 2010.

Comfort, L. K. (1999). Shared Risk: Complex Systems In Seismic Response. Amsterdam: Pergamon.

Comfort, L. K., Ko, K., \& Zagorecki, A. (2004). Coordination in Rapidly Evolving Disaster Response Systems The Role of Information. American Behavioral Scientist, 48(3), 295313.

Deutsche Presse-Agentur. (2011). Poor communication holds up aid to Japan after quake and tsunami. Retrieved September 25, 2012, from http://www.monstersandcritics.com/news/asiapacific/news/article_1629334.php/Poorcommunication-holds-up-aid-to-Japan-after-quake-and-tsunami.

Dmitracova, O. (2010). Poor coordination biggest problem for relief work - report. Retrieved September 25, 2012, from http://www.trust.org/alertnet/news/poor-coordinationbiggest-problem-for-relief-work-report-.

Engelmann, H., \& Fiedrich, F. (2007). Decision Support for the Members of an Emergency Operation Centre after an Earthquake. In B. Van de Walle, P. Burghardt, \& C. Nieuwenhuis (Eds.), Proceedings of the 4th International Conference on Information Systems for Crisis Response and Management (ISCRAM) - Delft, The Netherlands. May 13-16 2007.

FEMA (2011). FEMA Disasters \& Maps. Retrieved September 25, 2012, from http://www.mmrs.fema.gov/hazard/index.shtm.

Fiedrich, F., Gehbauer, F., and Rickers, U. (2000). Optimized resource allocation for emergency response after earthquake disasters. Safety Science, 35(1-3), 41-57.

IFRC. Disaster management - IFRC. Retrieved September 25, 2012, from http://www.ifrc.org/en/what-we-do/disaster-management/.

Klingner, B. (2011). Fukushima Crisis Shows Weakness in Japanese Crisis Management. Retrieved September 25, 2012, from http://www.heritage.org/research/commentary/2011/10/fukushima-crisis-showsweakness-in-japanese-crisis-management.

Klir, G. J., \& Yuan, B. (1995). Fuzzy sets and fuzzy logic: Theory and applications. Upper Saddle River, N.J., USA: Prentice Hall PTR.

Krolicki, K. (2011). Special Report: Mistakes, misfortune, meltdown: Japan's quake. Retrieved September 25, 2012, from http://www.reuters.com/article/2011/03/17/usjapan-quake-meltdown-specialreport-idUSTRE72G65Z20110317.

Nair, R., Ito, T., Tambe, M., \& Marsella, S. (2002). Task Allocation in the RoboCup Rescue Simulation Domain: A Short Note. Lecture Notes in Computer Science, 2002 (2377), 751-754.

New South Wales Government. Emergency Operations. Retrieved September 25, 2012, from http://www.ambulance.nsw.gov.au/about-us/Emergency-Operations.html.

Ramchurn, S. D., Rogers, A., Macarthur, K., Farinelli, A., Vytelingum, P., Vetsikas, I., \& Jennings, N. R. (2008). Agent-based coordination technologies in disaster management. In L. Padgham, D.C. Parkes, J.P. Müller, \& S. Parsons (Eds.), Proceedings of the 7th 
International Joint Conference on Autonomous Agents and Multiagent Systems (pp. 1651-1652). Estoril, Portugal, May 12 - 16, 2008.

Reijers, H. A., Jansen-Vullers, M. H., Zur Muehlen, M., \& Appl, W. (2007). Workflow management systems + swarm intelligence $=$ dynamic task assignment for emergency management applications. In G. Alonso, P. Dadam, \& M. Rosemann (Eds.), Proceedings of the 5th International Conference on Business Process Management, 2007 (pp. 125-140). Brisbane, Australia, September 24-28, 2007. LNCS 4714, Berlin, Heidelberg, New York: Springer.

Rolland, E., Patterson, R., Ward, K., \& Dodin, B. (2010). Decision support for disaster management. Operations Management Research, 3(1), 68-79.

Sanders, S. (2011). Japan's Sendai earthquake: One month later. The Washington Post. Retrieved September 25, 2012, from

http://www.washingtonpost.com/blogs/blogpost/post/japans-sendai-earthquake-onemonth-later/2011/04/11/AFdrZtLD_blog.html.

Shapiro, S. S., \& Wilk, M. B. (1965). An Analysis of Variance Test for Normality (Complete Samples). Biometrika, 52(3/4), 591-611.

Sharda, R., Barr, S.H., \& McDonnell, J.C. (1988). Decision Support System Effectiveness: A Review and an Empirical Test. Management Science, 34(2), 139-159.

U.S. Department of Homeland Security (2008). Homeland Security Advisory System-Guidance for Federal Departments and Agencies. Retrieved September 25, 2012, from www.westgov.org/component/joomdoc/doc_download/281-department-of-homelandsecurity-national-security-threat-levels-

unclassified\&ei=dophunuzli724qta04cqaw\&usg=afqjengaanrxxgvzqvwubvrfmb5v18w pbw\&sig2 $=$ mkpetvsvh2srsw6ex4tqnq.

Weng, M. X., Lu, J., \& Ren, H. (2001). Unrelated parallel machine scheduling with setup consideration and a total weighted completion time objective. International Journal of Production Economics, 70 (3), 215-226.

Wex, F., Schryen, G., \& Neumann, D. (2011). Intelligent Decision Support for Centralized Coordination during Emergency Response. In M.A. Santos, L. Sousa, \& E. Portela (Eds.), Proceedings of the 8th International Conference on Information Systems for Crisis Response and Management (ISCRAM). Lisbon, Portugal, May 8-11, 2011.

Wex, F., Schryen, G., \& Neumann, D. (2012). Operational Emergency Response under Informational Uncertainty: A Fuzzy Optimization Model for Scheduling and Allocating Rescue Units. In L. Rothkrantz, J. Ristvej, \& Z. Franco (Eds.), Proceedings of the 9th International Conference on Information Systems for Crisis Response and Management (ISCRAM). Vancouver, Canada, April 22-25, 2012.

Zadeh, L. A. (1965). Fuzzy sets. Information and Control, 8(3), 338-353.

Zimmermann, H. J. (2000). An application-oriented view of modeling uncertainty. European Journal of Operational Research, 122(2), 190-198. 


\section{Appendix}

\section{Proof of NP-hardness}

The RUASP (M1) is a generalization of the machine scheduling problem "Identical parallel machine non-preemptive scheduling with minimization of sum of completion times" (M2), which is NP-hard (Blazewicz et al., 1991): if we map incidents on jobs and rescue agents on machines, then the generalization refers to the fact that our problem provides for setup times (travel times), non-identical machines, and constraints on the assignment of rescue units to incidents. Given an instance of M2, we can map this instance onto an instance of M1 (in polynomial time) by ignoring each parameter that belongs to a fuzzy set, by setting $\widetilde{s_{l j}^{k}}=0$ for all jobs $\mathrm{i}, \mathrm{j}$ and for all machines $\mathrm{k}$, by setting $\widetilde{p_{l}^{k_{1}}}=\widetilde{p_{l}^{k_{2}}}$ for all jobs $\mathrm{i}$ and all machines $\mathrm{k}_{1}$ and $\mathrm{k}_{2}$, and by setting $c a p_{k l}=1$ for all rescue units $\mathrm{k}$ and for all incidents $\mathrm{i}$. Thus, our problem is NP-hard, too. Integrating Fuzzy Set Theory in this proof even raises the complexity.

\section{Pseudocode of the Monte Carlo Heuristic}

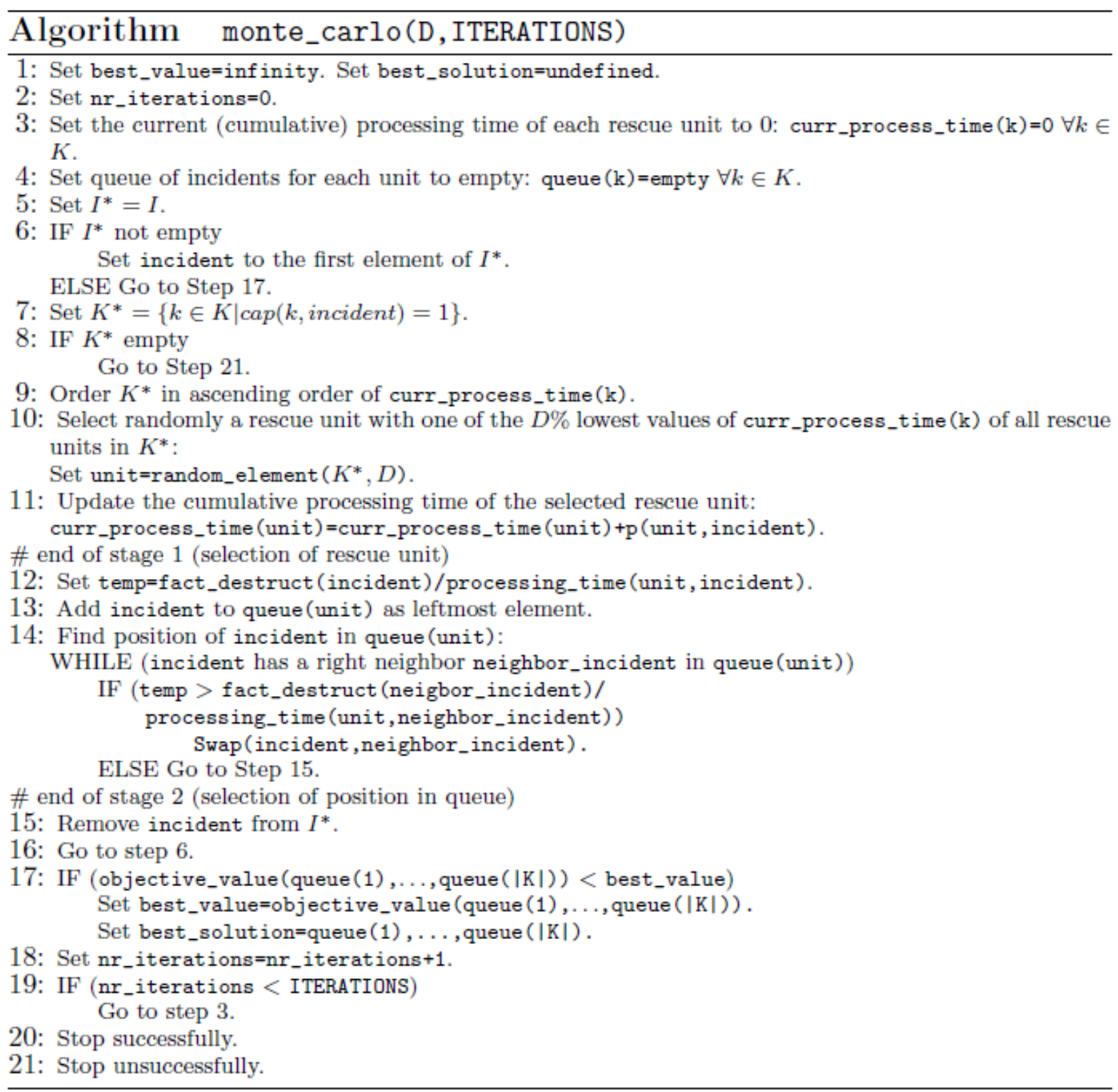


Felix Wex is a PhD student and research associate at the Chair of Information Systems Research at the University of Freiburg (Germany). He holds a Diploma in Information Engineering and Management from the Karlsruhe Institute of Technology (Germany). His research focusses on decision support systems with particular emphasis on crisis management. From November 2011 to January 2012, Felix Wex joined the Kitamoto Laboratory at the National Institute of Informatics in Tokyo (Japan) for a research visit.

Trained in computer science, information systems and operations research at RWTH Aachen University (Germany), Guido Schryen works in the field of decision support systems. After research visits to Stanford University and Queensland University of Technology (Brisbane, Australia), he received a doctorate in information systems from the RWTH Aachen University and earned a post-doctorate degree (habilitation/venia legendi) at the RWTH Aachen University. From May 2007 to March 2011, he held a position as Assistant Professor at the Institute of Business Information Systems, RWTH Aachen University. From September 2008 to August 2009, he joined the International Computer Science Institute (ICSI) at Berkeley (USA). From October 2009 to July 2010 and from October 2010 to March 2011 he was temporary professor of Information Systems Research at the University of Freiburg and at the University of Kiel (Germany), respectively. Since April 2011, Guido Schryen is professor of Information Systems Research at the University of Regensburg (Germany).

Prof. Dirk Neumann is a Full Professor with the Chair of Information Systems Research of the University of Freiburg (Germany). His research topics include Business Analytics, Cloud Computing, Green IT, Smart Grids, Emergency Response Systems and Green Logistics. He studied Information systems in Giessen (Diploma), Economics in Milwaukee, WI, USA (Master), and received a PhD from Karlsruhe in 2004. In 2011 he was awarded with the IBM faculty award for his research in the area of Big Data Analytics. 